\title{
Electric field controlled Faraday rotation in an electro-optic/magneto-optic bilayer
}

\author{
Yu. S. Dadoenkova, ${ }^{1, a)}$ I. L. Lyubchanskii, ${ }^{1, b)}$ Y. P. Lee, ${ }^{2, c)}$ and Th. Rasing ${ }^{3, d)}$ \\ ${ }^{1}$ Donetsk Physical and Technical Institute of the NAS of Ukraine, 83114 Donetsk, Ukraine, and Department \\ of Physics, Donetsk National University, 83000 Donetsk, Ukraine \\ ${ }^{2}$ Quantum Photonic Science Research Center ( $\left.q-P s i\right)$ and Department of Physics, Hanyang University, \\ Seoul 133-791, Republic of Korea \\ ${ }^{3}$ Institute for Molecules and Materials, Radboud University Nijmegen, 6525 AJ Nijmegen, The Netherlands
}

(Received 12 May 2010; accepted 2 June 2010; published online 6 July 2010; publisher error corrected 30 August 2010)

The Faraday rotation for light transmission through a bilayered structure composed of an electro-optic semiconductor film and a slab with high values of magneto-optic and magnetoelectric interactions is theoretically investigated. The influence of dispersion as well as an external electric field on the Faraday rotation is studied. (C) 2010 American Institute of Physics.

[doi:10.1063/1.3456534]

The magneto-optical (MO) Faraday effect (FE) which is characterized by a rotation of the polarization plane, or Faraday rotation (FR), of the light transmitted through a magnetic material is a well known MO effect which is widely used for the investigation of thin magnetic films and magnetic multilayers. ${ }^{1,2}$ For practical reasons it is sometimes necessary to enhance the FR angle by external physical factors. An attractive method to control the FE is by a permanent or alternating electric field applied to a magnetic material. The influence of an electric field on the FR can be described in terms of linear and quadratic electro-optical (EO) interactions which are responsible for the Pockels and Kerr EO effects, respectively. ${ }^{3}$ The effect of an electric field on the FR in yttrium-iron garnet (YIG) $\mathrm{Y}_{3} \mathrm{Fe}_{5} \mathrm{O}_{12}$, the socalled electro-MO effect, was experimentally observed in Ref. 4. The value of the induced FR was approximately equal to $1 \mathrm{arc} \mathrm{sec}$ for an electric field strength of $8.5 \mathrm{MV} / \mathrm{m}$. ${ }^{4} \mathrm{An}$ other possibility to enhance the FR in magnetic media is to use nonlinear optical effects. An example is the nonlinear FE, i.e., an intensity-dependent Faraday rotation which is described by a cubic nonlinear MO susceptibility tensor. ${ }^{5}$ Theoretical estimations of this effect showed that for an electric field magnitude of the laser light of about $10 \mathrm{KV} / \mathrm{m}$, the FR angle should be about $0.3^{\circ}$ for a rare-earth orthoferrite. Experimental observation of the nonlinear FE in the semimagnetic semiconductor CdMnTe yielded approximately $0.2^{\circ}$ per $1 \mathrm{~mm}$ at a laser radiation intensity of $5 \mathrm{GW} / \mathrm{cm}^{2} .6$ These FR angles induced by MO interactions in applied static and alternating electric fields are relatively small, leading to problems for precise measurements and are not very promising for applications. An alternative route could be to use heterogeneous multilayered systems composed of an MO film and another material with high EO parameters.

In this letter, we investigate the influence of a static external electric field on the FR of complex magneticnonmagnetic materials, which are promising structures for spintronics.

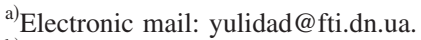

${ }^{b)}$ Electronic mail: igorl@fti.dn.ua.

${ }^{c)}$ Electronic mail: yplee@ hanyang.ac.kr.

${ }^{\mathrm{d})}$ Electronic mail: th.rasing@science.ru.nl.
}

Let us consider the transmission of a plane electromagnetic wave (EMW) through a bilayer structure consisting of an electro-optic film (EOF) and a magneto-optic film (MOF) of thicknesses $d_{\mathrm{EOF}}$ and $d_{\mathrm{MOF}}$, respectively, as shown in Fig. 1 . We assume the MOF is magnetized perpendicularly to the interface and is characterized by a magnetoelectric effect (ME). We shall study two cases of the polar MO configuration: the magnetization vector $\mathbf{M}$ is coincident with the propagation direction $z$ of the incident light $[\mathbf{M} \uparrow \uparrow O Z$, Fig. 1(a)] and $\mathbf{M}$ is antiparallel to the $z$-axis $[\mathbf{M} \uparrow \downarrow O Z$, Fig. 1(b)]. A permanent electric field $\mathbf{E}_{\mathbf{0}}=\left\{E_{0 x}, E_{0 y}, E_{0 z}\right\}$ is applied to the system described above in an arbitrary direction.

The electric and magnetic fields of the EMWs in the EOF and MOF are determined by the solutions of the Maxwell equations. ${ }^{8}$ The displacement vector $\mathbf{D}$ and the magnetic induction $\mathbf{B}$ in general case are connected to the electric field $\mathbf{E}$ and the magnetic field $\mathbf{H}$ of EMWs via material relations as follows: ${ }^{8,9}$

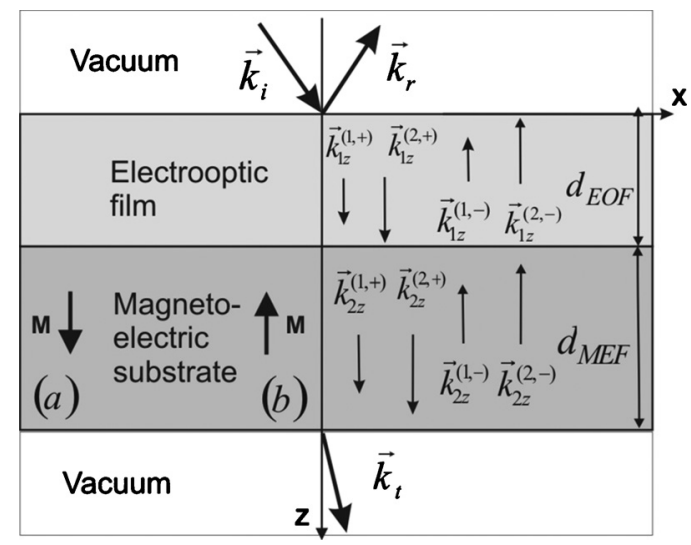

FIG. 1. Light transmission through an EO film on a MO slab: (a) $\mathbf{M} \uparrow \uparrow O Z$ and (b) $\mathbf{M} \uparrow \downarrow O Z$. Here $\mathbf{k}_{\mathbf{i}}, \mathbf{k}_{\mathbf{r}}$, and $\mathbf{k}_{\mathbf{t}}$ denote wave vectors of incident, reflected, and transmitted waves in vacuum, respectively, $k_{1 z}^{(1,+)}$ and $k_{1 z}^{(2,+)}$ $\left(k_{1 z}^{(1,-)}\right.$ and $\left.k_{1 z}^{(2,-)}\right)$ are z-components of wave vectors of transmitted (reflected) waves in the EOF, $k_{2 z}^{(1,+)}$ and $k_{2 z}^{(2,+)}\left(k_{2 z}^{(1,-)}\right.$ and $\left.k_{2 z}^{(2,-)}\right)$ denote $z$-components of the wave vectors of transmitted (reflected) waves in the MOF. 

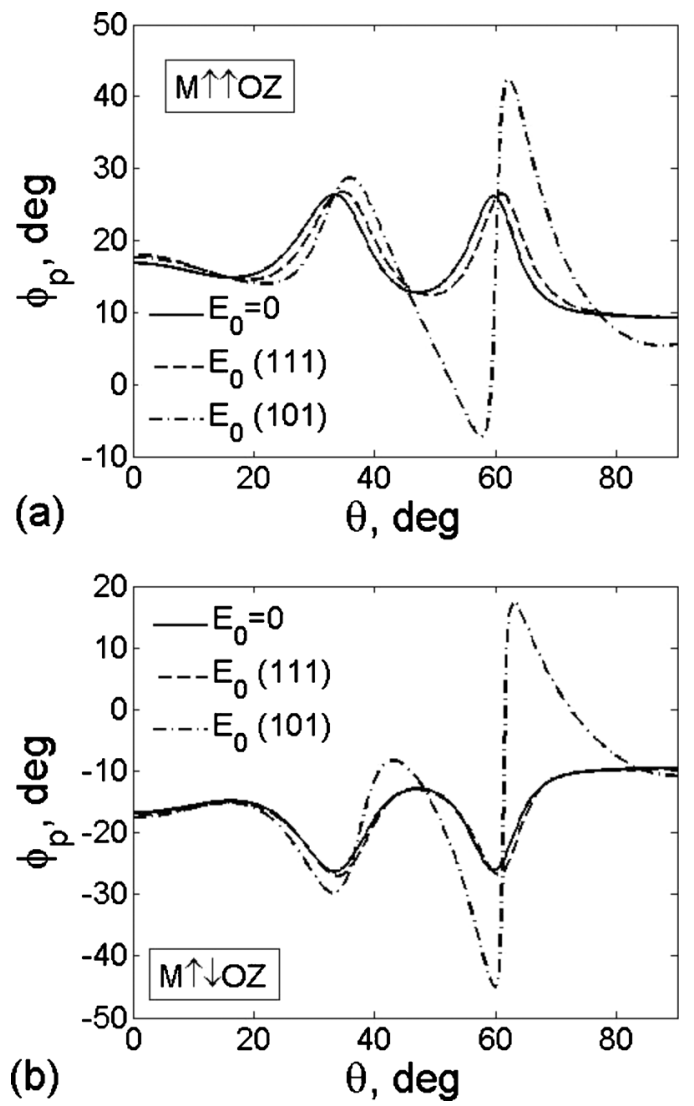

FIG. 2. FR angle $\phi_{p}^{F}$ as function of $\theta$ for fixed wavelength $\lambda=1.15 \mu \mathrm{m}$ for different orientations of the external electric field $\mathbf{E}_{0}$ and for two cases of the magnetization vector orientation: (a) $\mathbf{M} \uparrow \uparrow O Z$ and (b) $\mathbf{M} \uparrow \downarrow O Z$.

$$
D_{i}^{(n)}=\epsilon_{i j}^{(n)} E_{j}^{(n)}+\alpha_{i j}^{(n)} H_{j}^{(n)}, \quad B_{i}^{(n)}=\mu_{i j}^{(n)} H_{j}^{(n)}+\alpha_{j i}^{(n)} E_{j}^{(n)},
$$

where $\epsilon_{i j}^{(n)}, \mu_{i j}^{(n)}$, and $\alpha_{i j}^{(n)}$ are the permittivity, permeability, and linear ME tensors, respectively. Superscript $(n)$ denotes the EOF and MOF, respectively $(n=1,2)$.

The EO effects consist in a change in the optical properties of a material in response to an electric field. The tensor of the dielectric permittivity of the EO material $\epsilon_{i j}^{(1)}$ can be represented as a power series on the applied electric field strength $\mathbf{E}_{0}$ as follows: ${ }^{3}$

$$
\epsilon_{i j}^{(1)}=\epsilon_{0, i j}^{(1)}+r_{i j k} E_{0 k}+R_{i j k l} E_{0 k} E_{0 l}+\ldots,
$$

where $\epsilon_{0, i j}^{(1)}$ is the permittivity without external electric field and $r_{i j k}$ and $R_{i j k l}$ are linear and quadratic EO tensors, respectively. For a cubic crystal the tensor $\epsilon_{0, i j}^{(1)}$ has diagonal form $\epsilon_{0, i j}^{(1)}=\widetilde{\epsilon}^{(1)} \delta_{i j}$, where $\delta_{i j}$ is delta-symbol, and the form of the EO tensors can be found in Ref. 3. Tensors $r_{i j k}$ and $R_{i j k l}$ possess the following permutation properties: $r_{i j k}=r_{j i k}, R_{i j k l}$ $=R_{j i k l}$, and $R_{i j k l}=R_{i j l k} .{ }^{3}$ Nonzero components of $r_{i j k}$ and $R_{i j k l}$ can be found from the Tables 7.2 and 7.4 in Ref. 3. All components $\alpha_{i j}^{(1)}$ of the ME tensor are equal to zero for the EOF.

In our study, we consider the linear ME effect in the MOF and neglect the higher order ME coupling. The ME tensor $\hat{\alpha}^{(2)}$ for a cubic crystal has the diagonal form $\alpha_{i j}^{(2)}$ $=\alpha \delta_{i j}{ }^{10}$ It should be noted that the external electric field rotates the vector $\mathbf{M}$ over the value $\Delta M_{i}=\alpha_{i j} E_{0 j}$. Tensors $\hat{\boldsymbol{\epsilon}}^{(2)}$ and $\hat{\mu}^{(2)}$ can be expanded into series in the components of the unit magnetization vector $\mathbf{m}=\mathbf{M} /\left|\mathbf{M}_{s}\right|$, where $\mathbf{M}_{\mathbf{s}}$ is the saturation magnetization as follows: ${ }^{11}$
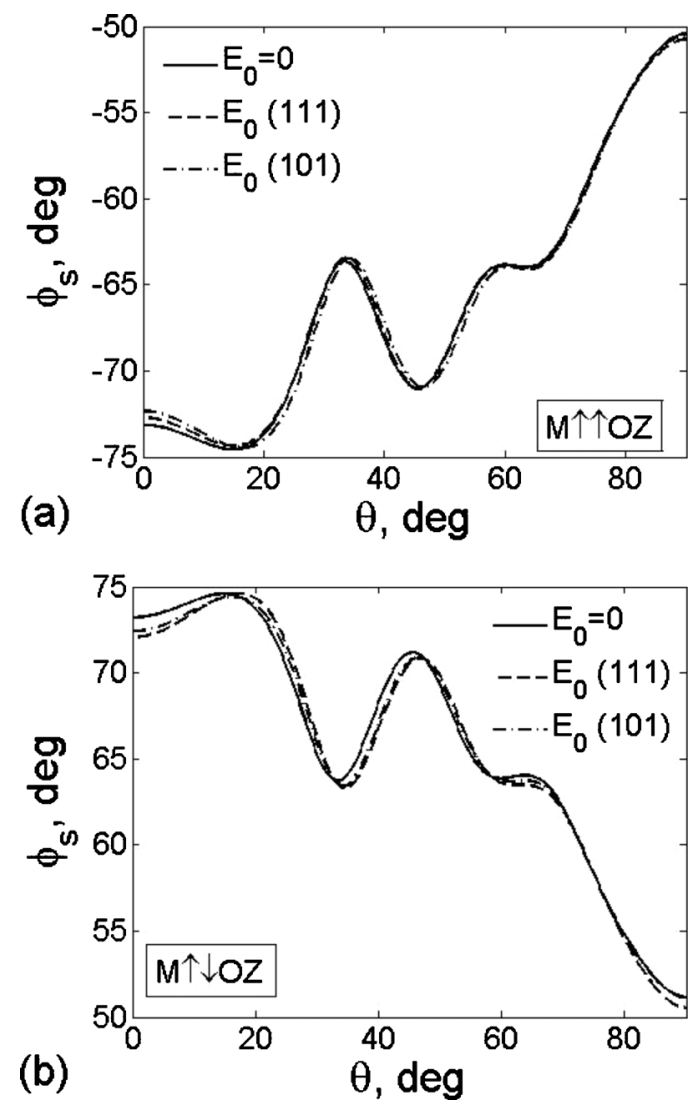

FIG. 3. FR angle $\phi_{s}^{F}$ as function of $\theta$ for fixed wavelength $\lambda=1.15 \mu \mathrm{m}$ for different orientations of the external electric field $\mathbf{E}_{\mathbf{0}}$ and for two cases of the magnetization vector orientation: (a) $\mathbf{M} \uparrow \uparrow O Z$ and (b) $\mathbf{M} \uparrow \downarrow O Z$.

$$
\begin{aligned}
& \epsilon_{i j}^{(2)}=\widetilde{\epsilon}^{(2)} \delta_{i j}+i f_{i j k}^{(e)} m_{k}+g_{i j k l}^{(e)} m_{k} m_{l}, \\
& \mu_{i j}^{(2)}=\tilde{\mu}^{(2)} \delta_{i j}+i f_{i j k}^{(m)} m_{k}+g_{i j k l}^{(m)} m_{k} m_{l},
\end{aligned}
$$

where $\widetilde{\epsilon}^{(2)}$ and $\widetilde{\mu}^{(2)}$ are the crystallographic components of the permittivity and permeability tensors, respectively, and $f_{i j k}^{(e, m)}$ and $g_{i j k l}^{(e, m)}$ denote MO tensors that are linear and quadratic in the magnetization. In Eqs. (3) and (4), we neglect the terms which describe the electro-MO effect in the MOF, because their contribution to the FR is very small. ${ }^{4}$

Dispersion relations of $\widetilde{\epsilon}^{(1)}$ and $\widetilde{\epsilon}^{(2)}$ can be obtained from the Sellmeier equation in the following form: ${ }^{12,13}$

$$
\widetilde{\epsilon}^{(n)}(\omega)=\varepsilon^{(n)}+\sum \frac{f_{i}^{(n)}}{1-\omega^{2} h_{i}^{(n)} /(2 \pi c)^{2}},
$$

where $\omega$ is the EMW's frequency, $\varepsilon^{(n)}, f_{i}^{(n)}$, and $h_{i}^{(n)}$ are the Sellmeier coefficients for the corresponding medium. ${ }^{12,13}$

After applying a standard method to solve the Maxwell equations ${ }^{1}$ we can write the transmitted light magnitudes $\mathbf{E}^{(\mathbf{t})}$ in terms of the incident light magnitudes $\mathbf{E}^{(\mathbf{i})}$ using the transmission matrix $\hat{T}$ as follows: ${ }^{2}$

$$
\left(\begin{array}{c}
E_{s}^{(t)} \\
E_{p}^{(t)}
\end{array}\right)=\hat{T}\left(\begin{array}{c}
E_{s}^{(i)} \\
E_{p}^{(i)}
\end{array}\right), \quad \hat{T}=\left(\begin{array}{cc}
T_{s s} & T_{s p} \\
T_{p s} & T_{p p}
\end{array}\right),
$$

where subscripts $p$ and $s$ correspond to the $p$-polarized and $s$-polarized EMWs, respectively.

The FR angle $\phi^{F}$ calculated from the ratio $\kappa$ of the complex $E_{s}^{(t)}$ and $E_{p}^{(t)}$ components of the transmitted field, $\kappa=E_{s}^{(t)} / E_{p}^{(t)}$, as 

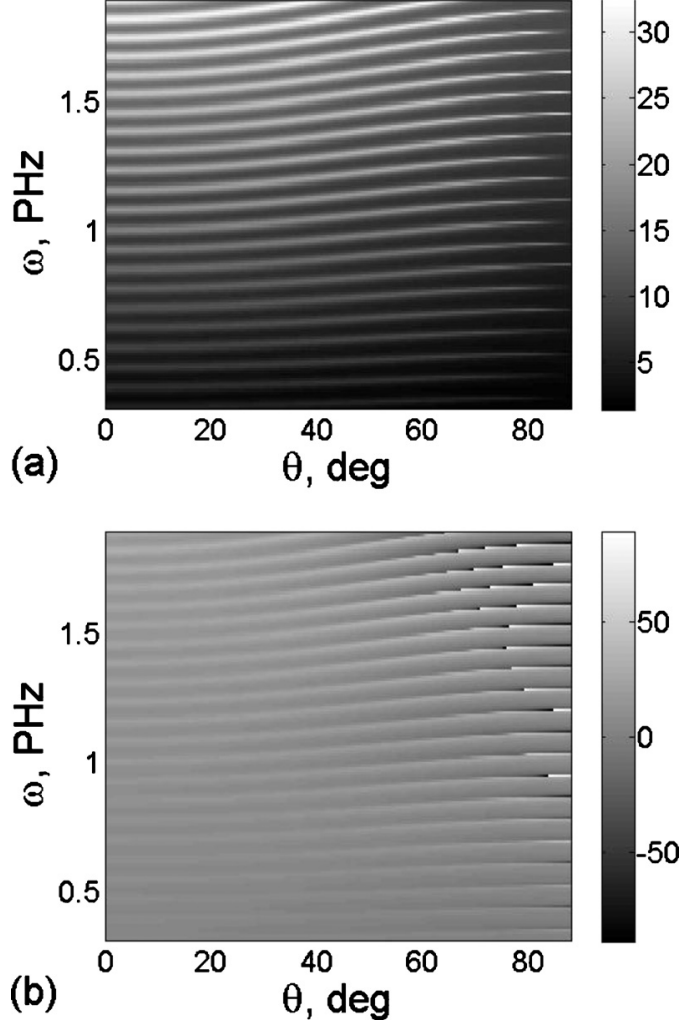

FIG. 4. Two-dimensional plot of the FR angle $\phi_{p}^{F}$ evolution with EMW's frequency $\omega$ and incidence angle $\theta$ for $\mathbf{M} \uparrow \uparrow O Z$ : (a) $\mathbf{E}_{\mathbf{0}}$ along (111) direction and (b) $\mathbf{E}_{\mathbf{0}}$ along (101) direction.

$$
\phi^{F}=\phi_{0}+\frac{1}{2} \operatorname{arctg}\left[2 \operatorname{Re}(\kappa) \times\left(1-|\kappa|^{2}\right)^{(-1)}\right],
$$

where $\phi_{0}=0$ for $|\kappa|^{2} \leq 1, \phi_{0}=\pi / 2$ for $|\kappa|^{2} \geq 1$ and $\operatorname{Re}(\kappa)$ $\geq 0$, and $\phi_{0}=-\pi / 2$ for $|\kappa|^{2} \geq 1$ and $\operatorname{Re}(\kappa) \leq 0$.

For the numerical calculations we consider a $\mathrm{ZnSe}$ semiconductor film as the EOF and an YIG film $\mathrm{Y}_{3} \mathrm{Fe}_{5} \mathrm{O}_{12}$ doped with $\mathrm{Bi}$ as the medium which demonstrates giant $\mathrm{MO}$ and ME interactions. ${ }^{14}$ In YIG the linear ME effect is observed in regions involving defects and at low temperatures, after cooling in simultaneously applied electric and magnetic fields. ${ }^{15}$ In epitaxial YIG films, the ME constant can reach a maximum value $\alpha \sim 10^{-2}$ at room temperature. ${ }^{16}$

For our calculations, we assume the external electric field to be of magnitude $E_{0}=10^{7} \mathrm{~V} / \mathrm{m}$. Thicknesses of the EOF and MOF are taken to be $d_{\mathrm{EOF}}=0.5 \mu \mathrm{m}$ and $d_{\mathrm{MOF}}$ $=5 \mu \mathrm{m}$, respectively.

The results of the numerical calculations of the FR angles are presented in Figs. 2-4.

Figures 2 and 3 present the FR angles $\phi_{p}^{F}$ for $p$ - and $\phi_{s}^{F}$ for $s$-polarized incident light, respectively, for a fixed wavelength $\lambda=1.15 \mu \mathrm{m}$ in the cases of $\mathbf{M} \uparrow \uparrow O Z$ [Figs. 2(a) and 3(a)] and $\mathbf{M} \uparrow \downarrow O Z$ [Figs. 2(b) and 3(b)] for different orientations of the external electric field $\mathbf{E}_{\mathbf{0}}$. Here solid lines correspond to the FR angles in the absence of $\mathbf{E}_{\mathbf{0}}$, dotted and dashed-dotted lines depict FR angles for $\mathbf{E}_{\mathbf{0}}$ applied in the (111) and (101) directions, respectively. From Figs. 2(a) and 2(b) one can see that the FR angles are strongly influenced by the orientation of $\mathbf{M}$, as well as by the applied field $\mathbf{E}_{\mathbf{0}}$. The FR has opposite directions in the cases of $\mathbf{M} \uparrow \uparrow O Z$ and $\mathbf{M} \uparrow \downarrow$ OZ. One can see from the Figs. 2(a) and 2(b) that applying $\mathbf{E}_{\mathbf{0}}$ in the (101) direction leads to an enhancement of $\phi_{p}^{F}$ for both orientations of $\mathbf{M}$ for $p$-polarized incident light. Thus, external electric field application leads to the possibility of controlling the light transmission as well as the FR. For $s$-polarized incident light the difference in $\phi_{s}^{F}$ for both $\mathbf{E}_{\mathbf{0}}$ directions is relatively small in comparison to the case of $p$-polarized incident light and is equal to approximately one percent from $\phi_{s}^{F}$ without $\mathbf{E}_{\mathbf{0}}$ (see Fig. 3).

Figure 4 presents two-dimensional plots of the FR angle as a function of the EMW's frequency $\omega$ and incidence angle $\theta$ for $\mathbf{M} \uparrow \uparrow O Z$ for $p$-polarized incident light in the case of $\mathbf{E}_{\mathbf{0}}$ along (111) [Fig. 4(a)] and (101) [Fig. 4(b)]. One can see that the FR angles demonstrate a stripe structure of minima and maxima at some frequency bands. Taking into account the frequency dispersion leads to minima shifts (about a few percents) toward higher frequencies.

In conclusion, we have shown that in an artificial ME system composed of an EOF and a MOF, the FR for $p$-polarized incident radiation is very sensitive to the permanent external field orientation as well as to the mutual orientation of the $\mathbf{M}$ and the EMW's propagation direction, whereas for $s$-polarized incident light the influence of $\mathbf{E}_{\mathbf{0}}$ on the FR is relatively small. Reversal of the magnetization vector changes the polarization plane rotation direction. Thus, by applying an external electric field we can control the values of the polarization plane rotation angles, which can be very useful for applications in devices for modern photonics.

This work was supported partly via project FP7-People2009-IRSES, No. 247556 (Y.S.D. and I.L.L.), partly by National Research Foundation (Korea) through q-Psi (Y.P.L.) and partly by NWO - the Netherlands Organization for Scientific Research (Th.R.).

${ }^{1}$ A. K. Zvezdin and V. A. Kotov, Modern Magneto-optics and Magnetooptical Materials (IOP, Bristol, 1997).

${ }^{2}$ M. Mansuripur, The Physical Principles of Magneto-optical Recording (Cambridge University Press, Cambridge, 1998).

${ }^{3}$ A. Yariv and P. Yeh, Optical Waves in Crystals (Wiley, New York, 1984).

${ }^{4}$ B. B. Krichevtsov, R. V. Pisarev, and A. G. Selitskii, JETP Lett. 41, 317 (1985).

${ }^{5}$ S. B. Borisov, I. L. Lyubchanskii, and A. D. Petrenko, Opt. Spectrosc. 64, 822 (1988).

${ }^{6}$ J. Frey, R. Frey, C. Flytzanis, and R. Triboulet, J. Opt. Soc. Am. B 9, 132 (1992)

${ }^{7}$ J. H. Versluis, A. V. Kimel, A. Kirilyuk, P. Grabs, F. Lehmann, G. Schmidt, L. W. Molenkamp, and Th. Rasing, Phys. Rev. B 80, 193303 (2009).

${ }^{8}$ M. Born and E. Wolf, Principles of Optics (Pergamon, London, 1997).

${ }^{9}$ T. H. O'Dell, The Electrodynamics of Magnetoelectric Media (NorthHolland, Amsterdam, 1970), p. 320.

${ }^{10}$ R. R. Birss, Symmetry and Magnetism (North-Holland, Amsterdam, 1966), p. 138.

${ }^{11}$ I. L. Lyubchanskii, N. N. Dadoenkova, M. I. Lyubchanskii, E. A. Shapovalov, A. Lakhtakia, and T. Rasing, Appl. Phys. Lett. 85, 5932 (2004).

${ }^{12} \mathrm{G}$. Ghosh, Handbook of Thermo-Optic Coefficients of Optical Materials with Applications (Academic, New York, 1998).

${ }^{13}$ Magnetic and Other Properties of Oxides and Related Compounds, Landolt-Börnstein, New Series, Group III Vol. 12, Pt. A, edited by K.-H. Hellewege and A. M. Hellewege (Springer, Berlin, 1978).

${ }^{14}$ R. V. Pisarev, B. B. Krichevtsov, V. V. Pavlov, and A. G. Selitsky, J. Magn. Soc. Jpn. 11, 33 (1987).

${ }^{15}$ H. Ogawa, E. Kita, Y. Mochida, K. Kohn, S. Kimura, A. Tasaki, and K. Siratori, J. Phys. Soc. Jpn. 56, 452 (1987).

${ }^{16}$ B. B. Krichevtsov, V. V. Pavlov, R. V. Pisarev, and A. G. Selitsky, Ferroelectrics 161, 65 (1994). 